\title{
7. Aboriginal and Torres Strait Islander Mothers and Babies
}

\section{Reporting of Aboriginality}

Maternal Aboriginality is under-reported on the PDC. One method of assessing the extent of under-reporting and monitoring changes over time is to compare the reporting of maternal Aboriginality to the PDC with reporting of maternal Aboriginality on birth registrations held by the NSW Registry of Births, Deaths and Marriages. Using capture-recapture methods, an estimate of the total number of babies born to Aboriginal or Torres Strait Islander mothers was obtained and compared with the number of babies born to Aboriginal or Torres Strait Islander mothers as reported to the PDC (Table 69). An estimate of the total number of babies born to Aboriginal or Torres Strait Islander mothers or fathers was also obtained and compared with the number of babies born to Aboriginal or Torres Strait Islander mothers as reported to the PDC (Table 70). The method used here is described in Chapter 3 (page 11).

For NSW overall, the estimated percentage of births to Aboriginal or Torres Strait Islander mothers reported to the PDC ranged from 63\% to $68 \%$ between 2006 and 2008. In 2008, reporting varied markedly between Local Health Districts, ranging from $14 \%$ in the Northern Sydney Local Health District to $93 \%$ in the Mid North Coast Local Health District, with reporting generally better in rural compared to urban areas (Table 69, Figure 2). Under-reporting means that numbers of births presented in this chapter should be interpreted with caution. The total number of babies born to Aboriginal or Torres Strait Islander mothers in 2008 is estimated to be 4,716 , about one and a half times higher than the number reported to the PDC.

Table 70 shows the number of babies born to Aboriginal or Torres Strait Islander mothers as reported to the PDC and the number of babies born to Aboriginal or Torres Strait Islander mothers or fathers as reported to the NSW Registry of Births, Deaths and Marriages between 2006 and 2008. Using capture-recapture methods, an estimate of the total number of babies born to Aboriginal or Torres Strait Islander mothers or fathers was obtained from both data sources. For NSW overall, the estimated percentage of births to Aboriginal or Torres Strait Islander mothers or fathers ranged from 44\% to 46\% between 2006 and 2008. In 2008, reporting ranged from $11 \%$ in the Northern Sydney Local Health District to $63 \%$ in the Western NSW and Far West Local Health Districts. There are therefore a substantial number of babies with nonAboriginal or Torres Strait Islander mothers and Aboriginal or Torres Strait Islander fathers who are not represented in the numbers reported in this chapter.

Table 69. Births to Aboriginal mothers by source of birth report, year of birth and Local Health District of residence, NSW 2006-2008\#

\begin{tabular}{|c|c|c|c|c|c|c|}
\hline $\begin{array}{l}\text { Year of birth- } \\
\text { Local Health District } \\
\text { of residence }\end{array}$ & $\begin{array}{c}\text { MDC } \\
\text { births } \\
\text { No. }\end{array}$ & $\begin{array}{c}\text { RBDM } \\
\text { births } \\
\text { No. }\end{array}$ & $\begin{array}{c}\text { Births reported } \\
\text { to both } \\
\text { MDC/RBDM } \\
\text { No. }\end{array}$ & $\begin{array}{c}\text { Total estimated } \\
\text { Aboriginal births } \\
\text { No. }\end{array}$ & $\begin{array}{c}\text { Estimated } \\
\text { Aboriginal births } \\
\text { reported to MDC } \\
\%\end{array}$ & $\begin{array}{l}95 \% \text { confidence } \\
\text { interval of } \\
\text { estimated births } \\
\text { reported }\end{array}$ \\
\hline \multicolumn{7}{|l|}{2006} \\
\hline Sydney & 99 & 115 & 64 & 177 & 55.8 & $48.5-63.1$ \\
\hline South Western Sydney & 118 & 215 & 72 & 351 & 33.6 & $28.7-38.5$ \\
\hline South Eastern Sydney & 74 & 99 & 52 & 141 & 52.7 & $44.4-60.9$ \\
\hline Illawarra Shoalhaven & 155 & 142 & 108 & 204 & 76.1 & $70.3-82.0$ \\
\hline Western Sydney & 194 & 209 & 123 & 329 & 58.9 & $53.6-64.2$ \\
\hline Nepean Blue Mountains & 91 & 93 & 56 & 151 & 60.4 & $52.6-68.2$ \\
\hline Northern Sydney & 16 & 38 & 6 & 94 & 17.1 & $9.5-24.7$ \\
\hline Central Coast & 88 & 106 & 64 & 146 & 60.5 & $52.5-68.4$ \\
\hline Hunter New England & 598 & 499 & 361 & 826 & 72.4 & $69.3-75.4$ \\
\hline Northern NSW & 203 & 143 & 115 & 252 & 80.5 & $75.6-85.4$ \\
\hline Mid North Coast & 158 & 87 & 75 & 183 & 86.3 & $81.3-91.3$ \\
\hline Southern NSW & 70 & 39 & 32 & 85 & 82.3 & $74.2-90.4$ \\
\hline Murrumbidgee & 121 & 88 & 66 & 161 & 75.1 & $68.5-81.8$ \\
\hline Western NSW & 527 & 380 & 330 & 607 & 86.9 & $84.2-89.5$ \\
\hline Far West & 44 & 34 & 31 & 48 & 91.3 & $83.3-99.2$ \\
\hline Other-not stated & 91 & 68 & 56 & 110 & 82.5 & $75.4-89.5$ \\
\hline NSW & 2647 & 2355 & 1611 & 3869 & 68.4 & $66.9-69.9$ \\
\hline \multicolumn{7}{|l|}{2007} \\
\hline Sydney & 97 & 126 & 58 & 210 & 46.2 & $39.5-52.9$ \\
\hline South Western Sydney & 160 & 237 & 87 & 434 & 36.8 & $32.3-41.4$ \\
\hline South Eastern Sydney & 86 & 106 & 33 & 273 & 31.5 & $26.0-37.0$ \\
\hline Illawarra Shoalhaven & 163 & 121 & 96 & 205 & 79.4 & $73.9-84.9$ \\
\hline Western Sydney & 221 & 222 & 118 & 415 & 53.3 & $48.5-58.1$ \\
\hline Nepean Blue Mountains & 144 & 124 & 81 & 220 & 65.4 & $59.2-71.7$ \\
\hline
\end{tabular}


Table 69. (Continued)

\begin{tabular}{|c|c|c|c|c|c|c|}
\hline $\begin{array}{l}\text { Year of birth- } \\
\text { Local Health District } \\
\text { of residence }\end{array}$ & $\begin{array}{c}\text { MDC } \\
\text { births } \\
\text { No. }\end{array}$ & $\begin{array}{c}\text { RBDM } \\
\text { births } \\
\text { No. }\end{array}$ & $\begin{array}{c}\text { Births reported } \\
\text { to both } \\
\text { MDC/RBDM } \\
\text { No. }\end{array}$ & $\begin{array}{c}\text { Total estimated } \\
\text { Aboriginal births } \\
\text { No. }\end{array}$ & $\begin{array}{c}\text { Estimated } \\
\text { Aboriginal births } \\
\text { reported to MDC } \\
\%\end{array}$ & $\begin{array}{l}95 \% \text { confidence } \\
\text { interval of } \\
\text { estimated births } \\
\text { reported }\end{array}$ \\
\hline Northern Sydney & 28 & 57 & 13 & 119 & 23.5 & $15.9-31.1$ \\
\hline Central Coast & 111 & 99 & 70 & 157 & 70.8 & $63.7-77.9$ \\
\hline Hunter New England & 661 & 506 & 383 & 873 & 75.7 & $72.9-78.6$ \\
\hline Northern NSW & 230 & 139 & 121 & 264 & 87.1 & $83.1-91.1$ \\
\hline Mid North Coast & 149 & 83 & 75 & 165 & 90.4 & $85.9-94.9$ \\
\hline Southern NSW & 67 & 37 & 31 & 80 & 84.0 & $76.0-92.1$ \\
\hline Murrumbidgee & 169 & 129 & 103 & 212 & 79.9 & $74.5-85.3$ \\
\hline Western NSW & 511 & 360 & 318 & 578 & 88.3 & $85.7-91.0$ \\
\hline Far West & 44 & 25 & 20 & 55 & 80.4 & $69.9-90.9$ \\
\hline Other-not stated & 82 & 68 & 51 & 109 & 75.1 & $67.0-83.2$ \\
\hline NSW & 2923 & 2439 & 1658 & 4300 & 68.0 & $66.6-69.4$ \\
\hline \multicolumn{7}{|l|}{2008} \\
\hline Sydney & 103 & 218 & 62 & 361 & 28.6 & $23.9-33.2$ \\
\hline South Western Sydney & 174 & 233 & 94 & 430 & 40.5 & $35.8-45.1$ \\
\hline South Eastern Sydney & 59 & 188 & 35 & 314 & 18.8 & $14.5-23.1$ \\
\hline Illawarra Shoalhaven & 196 & 146 & 123 & 233 & 84.3 & $79.6-89.0$ \\
\hline Western Sydney & 202 & 238 & 116 & 414 & 48.8 & $44.0-53.6$ \\
\hline Nepean Blue Mountains & 148 & 117 & 84 & 206 & 71.9 & $65.8-78.0$ \\
\hline Northern Sydney & 21 & 80 & 11 & 148 & 14.2 & 8.6-19.9 \\
\hline Central Coast & 123 & 114 & 81 & 173 & 71.1 & $64.4-77.9$ \\
\hline Hunter New England & 750 & 576 & 460 & 939 & 79.9 & $77.3-82.4$ \\
\hline Northern NSW & 203 & 139 & 114 & 247 & 82.1 & $77.3-86.9$ \\
\hline Mid North Coast & 172 & 94 & 87 & 186 & 92.6 & $88.8-96.4$ \\
\hline Southern NSW & 59 & 43 & 30 & 84 & 70.1 & $60.3-79.9$ \\
\hline Murrumbidgee & 150 & 106 & 80 & 198 & 75.6 & $69.6-81.6$ \\
\hline Western NSW & 535 & 328 & 285 & 616 & 86.9 & $84.2-89.6$ \\
\hline Far West & 53 & 27 & 24 & 59 & 89.1 & $81.2-97.0$ \\
\hline Other-not stated & 66 & 57 & 42 & 89 & 73.8 & $64.7-83.0$ \\
\hline NSW & 3014 & 2704 & 1728 & 4716 & 63.9 & $62.5-65.3$ \\
\hline
\end{tabular}

Figure 2. Level of reporting of maternal Aboriginality to the NSW Perinatal Data Collection by year of birth and Local Health District of residence, NSW 2008

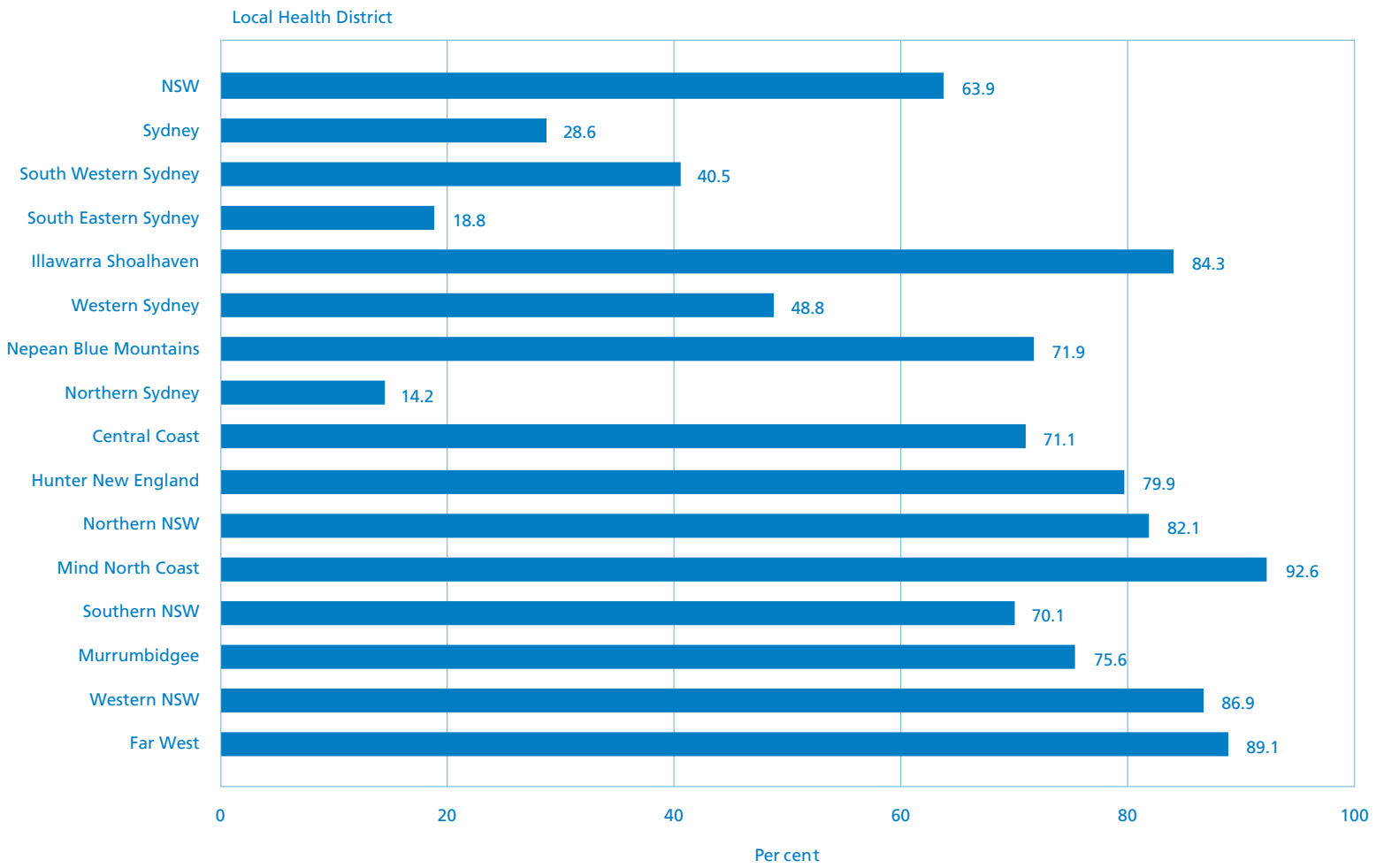

Source: Linked records of the NSW Perinatal Data Collection and Registry of Births, Deaths and Marriages birth registration data. 
Table 70. Births to Aboriginal mothers and fathers by source of birth report, year of birth and Local Health District of residence, NSW 2006-2008\#

\begin{tabular}{|c|c|c|c|c|c|c|}
\hline $\begin{array}{l}\text { Year of birth- } \\
\text { Local Health District } \\
\text { of residence }\end{array}$ & $\begin{array}{c}\text { MDC } \\
\text { births } \\
\text { No. }\end{array}$ & $\begin{array}{c}\text { RBDM } \\
\text { births } \\
\text { No. }\end{array}$ & $\begin{array}{c}\text { Births reported } \\
\text { to both } \\
\text { MDC/RBDM } \\
\text { No. }\end{array}$ & $\begin{array}{c}\text { Total estimated } \\
\text { Aboriginal births } \\
\text { No. }\end{array}$ & $\begin{array}{c}\text { Estimated } \\
\text { Aboriginal births } \\
\text { reported to MDC } \\
\%\end{array}$ & $\begin{array}{l}95 \% \text { confidence } \\
\text { interval of } \\
\text { estimated births } \\
\text { reported }\end{array}$ \\
\hline \multicolumn{7}{|l|}{2006} \\
\hline Sydney & 99 & 153 & 68 & 222 & 44.6 & $38.0-51.1$ \\
\hline South Western Sydney & 118 & 314 & 72 & 512 & 23.0 & $19.4-26.7$ \\
\hline South Eastern Sydney & 74 & 155 & 52 & 220 & 33.7 & $27.4-39.9$ \\
\hline Illawarra Shoalhaven & 155 & 239 & 110 & 336 & 46.1 & $40.8-51.4$ \\
\hline Western Sydney & 194 & 326 & 127 & 497 & 39.0 & $34.7-43.3$ \\
\hline Nepean Blue Mountains & 91 & 185 & 57 & 294 & 30.9 & $25.7-36.2$ \\
\hline Northern Sydney & 16 & 61 & 7 & 131 & 12.2 & $6.6-17.9$ \\
\hline Central Coast & 88 & 179 & 66 & 238 & 37.0 & $30.8-43.1$ \\
\hline Hunter New England & 598 & 835 & 372 & 1342 & 44.6 & $41.9-47.2$ \\
\hline Northern NSW & 203 & 212 & 116 & 370 & 54.8 & $49.7-59.9$ \\
\hline Mid North Coast & 158 & 136 & 76 & 282 & 56.0 & $50.3-61.8$ \\
\hline Southern NSW & 70 & 85 & 33 & 179 & 39.2 & $32.0-46.4$ \\
\hline Murrumbidgee & 121 & 145 & 69 & 253 & 47.7 & $41.6-53.9$ \\
\hline Western NSW & 527 & 516 & 332 & 819 & 64.4 & $61.1-67.6$ \\
\hline Far West & 44 & 50 & 31 & 71 & 62.2 & $50.9-73.5$ \\
\hline Other-not stated & 91 & 112 & 57 & 178 & 51.1 & $43.7-58.4$ \\
\hline NSW & 2647 & 3703 & 1645 & 5958 & 44.4 & $43.2-45.7$ \\
\hline \multicolumn{7}{|l|}{2007} \\
\hline Sydney & 97 & 165 & 64 & 249 & 38.9 & $32.9-45.0$ \\
\hline South Western Sydney & 160 & 352 & 91 & 617 & 25.9 & $22.5-29.4$ \\
\hline South Eastern Sydney & 86 & 148 & 34 & 369 & 23.3 & $19.0-27.6$ \\
\hline Illawarra Shoalhaven & 163 & 227 & 101 & 366 & 44.6 & $39.5-49.7$ \\
\hline Western Sydney & 221 & 341 & 123 & 611 & 36.2 & $32.3-40.0$ \\
\hline Nepean Blue Mountains & 144 & 203 & 83 & 351 & 41.0 & $35.9-46.2$ \\
\hline Northern Sydney & 28 & 86 & 13 & 179 & 15.6 & $10.3-20.9$ \\
\hline Central Coast & 111 & 177 & 73 & 268 & 41.4 & $35.5-47.2$ \\
\hline Hunter New England & 661 & 811 & 394 & 1360 & 48.6 & $46.0-51.3$ \\
\hline Northern NSW & 230 & 238 & 132 & 414 & 55.5 & $50.8-60.3$ \\
\hline Mid North Coast & 149 & 141 & 76 & 276 & 54.1 & $48.2-59.9$ \\
\hline Southern NSW & 67 & 67 & 33 & 135 & 49.6 & $41.2-58.1$ \\
\hline Murrumbidgee & 169 & 188 & 112 & 283 & 59.6 & $53.9-65.4$ \\
\hline Western NSW & 511 & 525 & 331 & 810 & 63.1 & $59.7-66.4$ \\
\hline Far West & 44 & 34 & 22 & 67 & 65.2 & $53.8-76.6$ \\
\hline Other-not stated & 82 & 109 & 54 & 165 & 49.7 & $42.1-57.3$ \\
\hline NSW & 2923 & 3812 & 1736 & 6418 & 45.5 & $44.3-46.8$ \\
\hline \multicolumn{7}{|l|}{2008} \\
\hline Sydney & 103 & 258 & 64 & 413 & 24.9 & $20.7-29.1$ \\
\hline South Western Sydney & 174 & 352 & 96 & 636 & 27.4 & $23.9-30.8$ \\
\hline South Eastern Sydney & 59 & 237 & 35 & 396 & 14.9 & $11.4-18.4$ \\
\hline Illawarra Shoalhaven & 196 & 236 & 128 & 361 & 54.3 & $49.2-59.4$ \\
\hline Western Sydney & 202 & 335 & 116 & 582 & 34.7 & $30.8-38.6$ \\
\hline Nepean Blue Mountains & 148 & 195 & 86 & 335 & 44.2 & $38.9-49.5$ \\
\hline Northern Sydney & 21 & 108 & 12 & 183 & 11.4 & $6.8-16.1$ \\
\hline Central Coast & 123 & 186 & 84 & 272 & 45.3 & $39.3-51.2$ \\
\hline Hunter New England & 750 & 865 & 464 & 1398 & 53.7 & $51.0-56.3$ \\
\hline Northern NSW & 203 & 233 & 122 & 387 & 52.4 & $47.5-57.4$ \\
\hline Mid North Coast & 172 & 159 & 91 & 300 & 57.4 & $51.8-63.0$ \\
\hline Southern NSW & 59 & 74 & 33 & 131 & 44.9 & $36.4-53.4$ \\
\hline Murrumbidgee & 150 & 178 & 87 & 306 & 49.0 & $43.4-54.6$ \\
\hline Western NSW & 535 & 475 & 299 & 849 & 63.0 & $59.7-66.2$ \\
\hline Far West & 53 & 40 & 25 & 84 & 63.0 & $52.7-73.3$ \\
\hline Other-not stated & 66 & 89 & 43 & 136 & 48.5 & $40.1-56.9$ \\
\hline NSW & 3014 & 4020 & 1785 & 6787 & 44.4 & $43.2-45.6$ \\
\hline
\end{tabular}




\section{Trends in births}

Between 2005 and 2009, the number of reported births to Aboriginal or Torres Strait Islander mothers rose from 2,507 to 2,931, representing $2.8 \%$ and $3.0 \%$ respectively of all babies born in NSW. In 2009, 2,784 babies were born to Aboriginal mothers, 45 babies were born to Torres Strait Islander mothers and 102 babies were born to mothers of both Aboriginal and Torres Strait Islander background (Table 71).

\section{Table 71. Aboriginal and Torres Strait Islander mothers and babies, NSW 2005-2009\#}

\begin{tabular}{|c|c|c|c|c|c|c|c|c|c|c|}
\hline \multirow{3}{*}{$\begin{array}{l}\text { Aboriginal and Torres Strait } \\
\text { Islander mothers and babies }\end{array}$} & \multicolumn{10}{|c|}{ Year } \\
\hline & \multicolumn{2}{|c|}{2005} & \multicolumn{2}{|c|}{2006} & \multicolumn{2}{|c|}{2007} & \multicolumn{2}{|c|}{2008} & \multicolumn{2}{|c|}{2009} \\
\hline & No. & $\%$ & No. & $\%$ & No. & $\%$ & No. & $\%$ & No. & $\%$ \\
\hline \multicolumn{11}{|l|}{ Mothers } \\
\hline Aboriginal but not Torres Strait Islander & 2347 & 94.9 & 2429 & 93.0 & 2700 & 93.5 & 2801 & 94.1 & 2757 & 94.9 \\
\hline Torres Strait Islander but not Aboriginal & 30 & 1.2 & 50 & 1.9 & 53 & 1.8 & 61 & 2.0 & 45 & 1.5 \\
\hline Both Aboriginal and Torres Strait Islander & 97 & 3.9 & 132 & 5.1 & 135 & 4.7 & 114 & 3.8 & 102 & 3.5 \\
\hline TOTAL & 2474 & 100.0 & 2611 & 100.0 & 2888 & 100.0 & 2976 & 100.0 & 2904 & 100.0 \\
\hline \multicolumn{11}{|l|}{ Babies } \\
\hline Aboriginal but not Torres Strait Islander & 2376 & 94.8 & 2463 & 93.0 & 2736 & 93.5 & 2836 & 94.1 & 2784 & 95.0 \\
\hline Torres Strait Islander but not Aboriginal & 31 & 1.2 & 51 & 1.9 & 54 & 1.8 & 61 & 2.0 & 45 & 1.5 \\
\hline Both Aboriginal and Torres Strait Islander & 100 & 4.0 & 135 & 5.1 & 136 & 4.6 & 118 & 3.9 & 102 & 3.5 \\
\hline TOTAL & 2507 & 100.0 & 2649 & 100.0 & 2926 & 100.0 & 3015 & 100.0 & 2931 & 100.0 \\
\hline
\end{tabular}

\section{Plurality}

Between 2005 and 2009 multiple pregnancies (twins, triplets etc.) were reported for about $1 \%$ of all pregnancies. In 2009,

$1.8 \%$ of babies born to Aboriginal or Torres Strait Islander mothers were twins (Table 72).

\section{Table 72. Aboriginal and Torres Strait Islander mothers and babies by plurality, NSW 2005-2009\#}

\begin{tabular}{|c|c|c|c|c|c|c|c|c|c|c|}
\hline \multirow[t]{3}{*}{ Plurality } & \multicolumn{10}{|c|}{ Year } \\
\hline & \multicolumn{2}{|c|}{2005} & \multicolumn{2}{|c|}{2006} & \multicolumn{2}{|c|}{2007} & \multicolumn{2}{|c|}{2008} & \multicolumn{2}{|c|}{2009} \\
\hline & No. & $\%$ & No. & $\%$ & No. & $\%$ & No. & $\%$ & No. & $\%$ \\
\hline \multicolumn{11}{|l|}{ Mothers } \\
\hline Singleton & 2443 & 98.7 & 2574 & 98.6 & 2851 & 98.7 & 2937 & 98.7 & 2877 & 99.1 \\
\hline Twins & 29 & 1.2 & 36 & 1.4 & 36 & 1.2 & 39 & 1.3 & 27 & 0.9 \\
\hline Triplets & 2 & 0.1 & 1 & 0.0 & 1 & 0.0 & 0 & 0.0 & 0 & 0.0 \\
\hline TOTAL & 2474 & 100.0 & 2611 & 100.0 & 2888 & 100.0 & 2976 & 100.0 & 2904 & 100.0 \\
\hline \multicolumn{11}{|l|}{ Babies } \\
\hline Singleton & 2443 & 97.4 & 2574 & 97.2 & 2851 & 97.4 & 2937 & 97.4 & 2877 & 98.2 \\
\hline Twins & 58 & 2.3 & 72 & 2.7 & 72 & 2.5 & 78 & 2.6 & 54 & 1.8 \\
\hline Triplets & 6 & 0.2 & 3 & 0.1 & 3 & 0.1 & 0 & 0.0 & 0 & 0.0 \\
\hline TOTAL & 2507 & 100.0 & 2649 & 100.0 & 2926 & 100.0 & 3015 & 100.0 & 2931 & 100.0 \\
\hline
\end{tabular}

Source: NSW Perinatal Data Collection (HOIST). Centre for Epidemiology and Research, NSW Ministry of Health.

\# Due to under-reporting of Aboriginality to the PDC, it is likely that the true numbers of mothers are about one-and-a-half times higher than shown, and the true numbers of babies are about two times higher than shown. 


\section{Previous pregnancies}

In 2009, one-third of Aboriginal or Torres Strait Islander mothers gave birth for the first time (Table 73), 57.7\% of mothers reported between one and four previous births and
$7.8 \%$ of mothers had previously given birth to 5 or more babies. This pattern has not changed substantially since 2005 .

Table 73. Previous pregancies among Aboriginal and Torres Strait Islander mothers, NSW 2005-2009\#

\begin{tabular}{|c|c|c|c|c|c|c|c|c|c|c|}
\hline \multirow[t]{3}{*}{ No. previous pregnancies (>20 weeks) } & \multicolumn{10}{|c|}{ Year } \\
\hline & \multicolumn{2}{|c|}{2005} & \multicolumn{2}{|c|}{2006} & \multicolumn{2}{|c|}{2007} & \multicolumn{2}{|c|}{2008} & \multicolumn{2}{|c|}{2009} \\
\hline & No. & $\%$ & No. & $\%$ & No. & $\%$ & No. & $\%$ & No. & $\%$ \\
\hline 0 & 787 & 31.8 & 844 & 32.3 & 952 & 33.0 & 986 & 33.1 & 1000 & 34.4 \\
\hline $1-4$ & 1472 & 59.5 & 1551 & 59.4 & 1683 & 58.3 & 1735 & 58.3 & 1677 & 57.7 \\
\hline $5+$ & 214 & 8.6 & 199 & 7.6 & 237 & 8.2 & 253 & 8.5 & 227 & 7.8 \\
\hline Not stated & 1 & 0.0 & 17 & 0.7 & 16 & 0.6 & 2 & 0.1 & 0 & 0.0 \\
\hline TOTAL & 2474 & 100.0 & 2611 & 100.0 & 2888 & 100.0 & 2976 & 100.0 & 2904 & 100.0 \\
\hline
\end{tabular}

\section{Maternal age}

The reported number of Aboriginal or Torres Strait Islander mothers giving birth has increased at all ages over recent years. The percentage of Aboriginal or Torres Strait Islander mothers who were teenagers fell from $20.4 \%$ in 2005 to $18.9 \%$ in 2009. The percentage of mothers giving birth at 35 years of age or more has been stable at about 9\% (Table 74).

\section{Table 74. Age of Aboriginal and Torres Strait Islander mothers, NSW 2005-2009\#}

\begin{tabular}{|c|c|c|c|c|c|c|c|c|c|c|}
\hline \multirow[t]{3}{*}{ Maternal age (years) } & \multicolumn{10}{|c|}{ Year } \\
\hline & \multicolumn{2}{|c|}{2005} & \multicolumn{2}{|c|}{2006} & \multicolumn{2}{|c|}{2007} & \multicolumn{2}{|c|}{2008} & \multicolumn{2}{|c|}{2009} \\
\hline & No. & $\%$ & No. & $\%$ & No. & $\%$ & No. & $\%$ & No. & $\%$ \\
\hline $12-19$ & 504 & 20.4 & 534 & 20.5 & 543 & 18.8 & 589 & 19.8 & 549 & 18.9 \\
\hline $20-34$ & 1739 & 70.3 & 1851 & 70.9 & 2080 & 72.0 & 2109 & 70.9 & 2074 & 71.4 \\
\hline $35+$ & 231 & 9.3 & 222 & 8.5 & 264 & 9.1 & 278 & 9.3 & 281 & 9.7 \\
\hline Not stated & 0 & 0.0 & 4 & 0.2 & 1 & 0.0 & 0 & 0.0 & 0 & 0.0 \\
\hline TOTAL & 2474 & 100.0 & 2611 & 100.0 & 2888 & 100.0 & 2976 & 100.0 & 2904 & 100.0 \\
\hline
\end{tabular}




\section{Local Health District of residence}

Between 2005 and 2009, the reported number of Aboriginal or Torres Strait Islander mothers rose in most Local Health Districts. The reported number of Aboriginal of Torres Strait Islander mothers who gave birth in 2009 ranged from 28 in the Northern Sydney Local Health District to 654 in the Hunter
New England Local Health District (Table 75). The proportion of mothers who were teenagers varied from $15.3 \%$ in the Northern NSW Local Health District to $28.3 \%$ in the Nepean Blue Mountains Local Health District (Table 76).

Table 75. Local Health District of residence of Aboriginal and Torres Strait Islander mothers, NSW 2005-2009\#

\begin{tabular}{|c|c|c|c|c|c|c|c|c|c|c|}
\hline \multirow[t]{3}{*}{ Local Health District } & \multicolumn{10}{|c|}{ Year } \\
\hline & \multicolumn{2}{|c|}{2005} & \multicolumn{2}{|c|}{2006} & \multicolumn{2}{|c|}{2007} & \multicolumn{2}{|c|}{2008} & \multicolumn{2}{|c|}{2009} \\
\hline & No. & $\%$ & No. & $\%$ & No. & $\%$ & No. & $\%$ & No. & $\%$ \\
\hline Sydney & 74 & 3.0 & 97 & 3.7 & 96 & 3.3 & 102 & 3.4 & 105 & 3.6 \\
\hline South Western Sydney & 106 & 4.3 & 117 & 4.5 & 157 & 5.4 & 173 & 5.8 & 170 & 5.9 \\
\hline South Eastern Sydney & 70 & 2.8 & 69 & 2.6 & 85 & 2.9 & 57 & 1.9 & 64 & 2.2 \\
\hline Illawarra Shoalhaven & 124 & 5.0 & 151 & 5.8 & 162 & 5.6 & 190 & 6.4 & 170 & 5.9 \\
\hline Western Sydney & 180 & 7.3 & 191 & 7.3 & 221 & 7.7 & 201 & 6.8 & 203 & 7.0 \\
\hline Nepean Blue Mountains & 87 & 3.5 & 87 & 3.3 & 141 & 4.9 & 140 & 4.7 & 113 & 3.9 \\
\hline Northern Sydney & 17 & 0.7 & 17 & 0.7 & 27 & 0.9 & 21 & 0.7 & 28 & 1.0 \\
\hline Central Coast & 71 & 2.9 & 88 & 3.4 & 109 & 3.8 & 122 & 4.1 & 110 & 3.8 \\
\hline Hunter New England & 603 & 24.4 & 589 & 22.6 & 647 & 22.4 & 730 & 24.5 & 654 & 22.5 \\
\hline Northern NSW & 172 & 7.0 & 205 & 7.9 & 228 & 7.9 & 210 & 7.1 & 203 & 7.0 \\
\hline Mid North Coast & 168 & 6.8 & 190 & 7.3 & 195 & 6.8 & 203 & 6.8 & 234 & 8.1 \\
\hline Southern NSW & 73 & 3.0 & 71 & 2.7 & 66 & 2.3 & 58 & 1.9 & 68 & 2.3 \\
\hline Murrumbidgee & 143 & 5.8 & 128 & 4.9 & 178 & 6.2 & 152 & 5.1 & 156 & 5.4 \\
\hline Western NSW & 511 & 20.7 & 521 & 20.0 & 499 & 17.3 & 526 & 17.7 & 553 & 19.0 \\
\hline Far West & 42 & 1.7 & 43 & 1.6 & 43 & 1.5 & 51 & 1.7 & 35 & 1.2 \\
\hline Other-not stated & 33 & 1.3 & 47 & 1.8 & 34 & 1.2 & 40 & 1.3 & 38 & 1.3 \\
\hline TOTAL & 2474 & 100.0 & 2611 & 100.0 & 2888 & 100.0 & 2976 & 100.0 & 2904 & 100.0 \\
\hline
\end{tabular}

Table 76. Local Health District of residence of Aboriginal and Torres Strait Islander mothers by age, NSW 2009\#

\begin{tabular}{|c|c|c|c|c|c|c|}
\hline \multirow[t]{3}{*}{ Local Health District } & \multicolumn{6}{|c|}{ Maternal age (years) } \\
\hline & \multicolumn{2}{|c|}{ Under 20} & \multicolumn{2}{|c|}{ 20-plus } & \multicolumn{2}{|c|}{ TOTAL } \\
\hline & No. & $\%$ & No. & $\%$ & No. & $\%$ \\
\hline Sydney & 19 & 18.1 & 86 & 81.9 & 105 & 100.0 \\
\hline South Western Sydney & 38 & 22.4 & 132 & 77.6 & 170 & 100.0 \\
\hline South Eastern Sydney & 10 & 15.6 & 54 & 84.4 & 64 & 100.0 \\
\hline Illawarra Shoalhaven & 32 & 18.8 & 138 & 81.2 & 170 & 100.0 \\
\hline Western Sydney & 41 & 20.2 & 162 & 79.8 & 203 & 100.0 \\
\hline Nepean Blue Mountains & 32 & 28.3 & 81 & 71.7 & 113 & 100.0 \\
\hline Northern Sydney & - & - & - & - & 28 & 100.0 \\
\hline Central Coast & 17 & 15.5 & 93 & 84.5 & 110 & 100.0 \\
\hline Hunter New England & 128 & 19.6 & 526 & 80.4 & 654 & 100.0 \\
\hline Northern NSW & 31 & 15.3 & 172 & 84.7 & 203 & 100.0 \\
\hline Mid North Coast & 45 & 19.2 & 189 & 80.8 & 234 & 100.0 \\
\hline Southern NSW & 13 & 19.1 & 55 & 80.9 & 68 & 100.0 \\
\hline Murrumbidgee & 30 & 19.2 & 126 & 80.8 & 156 & 100.0 \\
\hline Western NSW & 103 & 18.6 & 450 & 81.4 & 553 & 100.0 \\
\hline Far West & 7 & 20.0 & 28 & 80.0 & 35 & 100.0 \\
\hline Other-not stated & - & - & - & - & 38 & 100.0 \\
\hline TOTAL & 549 & 18.9 & 2355 & 81.1 & 2904 & 100.0 \\
\hline
\end{tabular}




\section{Duration of pregnancy at first antenatal visit}

Between 2005 and 2009, the proportion of Aboriginal or Torres Strait Islander mothers who commenced antenatal care at less than 14 weeks gestation rose from $56.4 \%$ to $69.2 \%$, and the proportion who commenced antenatal care at less than 20 weeks gestation rose from $74.9 \%$ to $83.4 \%$ (Table 77). This compares with $79.2 \%$ of non-Aboriginal or Torres Strait Islander mothers who commenced antenatal care at less than 14 weeks gestation, and 89.2\% who commenced antenatal care at less than 20 weeks gestation in 2009.

In 2009, the proportion of Aboriginal or Torres Strait Islander mothers who commenced antenatal care at less than 14 weeks gestation varied from $34.3 \%$ in the Sydney Local Health District to $86.4 \%$ in the Central Coast Local Health District (Table 78).

Table 77. Duration of pregnancy at first antenatal visit among Aboriginal and Torres Strait Islander mothers, NSW 2005-2009\#

\begin{tabular}{|c|c|c|c|c|c|c|c|c|c|c|}
\hline \multirow[t]{3}{*}{ Duration of pregnancy (weeks) } & \multicolumn{10}{|c|}{ Year } \\
\hline & \multicolumn{2}{|c|}{2005} & \multicolumn{2}{|c|}{2006} & \multicolumn{2}{|c|}{2007} & \multicolumn{2}{|c|}{2008} & \multicolumn{2}{|c|}{2009} \\
\hline & No. & $\%$ & No. & $\%$ & No. & $\%$ & No. & $\%$ & No. & $\%$ \\
\hline $0-13$ & 1396 & 56.4 & 1531 & 58.6 & 1840 & 63.7 & 2045 & 68.7 & 2011 & 69.2 \\
\hline $14-19$ & 458 & 18.5 & 421 & 16.1 & 456 & 15.8 & 437 & 14.7 & 410 & 14.1 \\
\hline 20-plus & 561 & 22.7 & 550 & 21.1 & 461 & 16.0 & 404 & 13.6 & 428 & 14.7 \\
\hline Not stated & 59 & 2.4 & 109 & 4.2 & 131 & 4.5 & 90 & 3.0 & 55 & 1.9 \\
\hline TOTAL & 2474 & 100.0 & 2611 & 100.0 & 2888 & 100.0 & 2976 & 100.0 & 2904 & 100.0 \\
\hline
\end{tabular}

Table 78. Duration of pregnancy at first antenatal visit among Aboriginal and Torres Strait Islander mothers by Local Health District of residence, NSW 2009\#

\begin{tabular}{|c|c|c|c|c|c|c|c|c|c|c|}
\hline \multirow[t]{3}{*}{ Local Health District } & \multicolumn{10}{|c|}{ Duration of pregnancy (weeks) } \\
\hline & \multicolumn{2}{|c|}{$0-13$} & \multicolumn{2}{|c|}{$14-19$} & \multicolumn{2}{|c|}{ 20-plus } & \multicolumn{2}{|c|}{ Not stated } & \multicolumn{2}{|c|}{ TOTAL } \\
\hline & No. & $\%$ & No. & $\%$ & No. & $\%$ & No. & $\%$ & No. & $\%$ \\
\hline Sydney & 36 & 34.3 & 27 & 25.7 & 35 & 33.3 & 7 & 6.7 & 105 & 100.0 \\
\hline South Western Sydney & 87 & 51.2 & 36 & 21.2 & 44 & 25.9 & 3 & 1.8 & 170 & 100.0 \\
\hline South Eastern Sydney & 37 & 57.8 & 20 & 31.3 & 6 & 9.4 & 1 & 1.6 & 64 & 100.0 \\
\hline Illawarra Shoalhaven & 127 & 74.7 & 22 & 12.9 & 21 & 12.4 & 0 & 0.0 & 170 & 100.0 \\
\hline Western Sydney & 171 & 84.2 & 8 & 3.9 & 20 & 9.9 & 4 & 2.0 & 203 & 100.0 \\
\hline Nepean Blue Mountains & 91 & 80.5 & 10 & 8.8 & 10 & 8.8 & 2 & 1.8 & 113 & 100.0 \\
\hline Northern Sydney & 24 & 85.7 & - & - & - & - & 0 & 0.0 & 28 & 100.0 \\
\hline Central Coast & 95 & 86.4 & 8 & 7.3 & 6 & 5.5 & 1 & 0.9 & 110 & 100.0 \\
\hline Hunter New England & 463 & 70.8 & 88 & 13.5 & 93 & 14.2 & 10 & 1.5 & 654 & 100.0 \\
\hline Northern NSW & 150 & 73.9 & 25 & 12.3 & 22 & 10.8 & 6 & 3.0 & 203 & 100.0 \\
\hline Mid North Coast & 155 & 66.2 & 40 & 17.1 & 34 & 14.5 & 5 & 2.1 & 234 & 100.0 \\
\hline Southern NSW & 43 & 63.2 & 12 & 17.6 & 13 & 19.1 & 0 & 0.0 & 68 & 100.0 \\
\hline Murrumbidgee & 103 & 66.0 & 25 & 16.0 & 27 & 17.3 & 1 & 0.6 & 156 & 100.0 \\
\hline Western NSW & 380 & 68.7 & 76 & 13.7 & 83 & 15.0 & 14 & 2.5 & 553 & 100.0 \\
\hline Far West & 19 & 54.3 & 8 & 22.9 & 8 & 22.9 & 0 & 0.0 & 35 & 100.0 \\
\hline Other-not stated & 30 & 78.9 & - & - & - & - & 1 & 2.6 & 38 & 100.0 \\
\hline TOTAL & 2011 & 69.2 & 410 & 14.1 & 428 & 14.7 & 55 & 1.9 & 2904 & 100.0 \\
\hline
\end{tabular}

Source: NSW Perinatal Data Collection (HOIST). Centre for Epidemiology and Research, NSW Ministry of Health.

\# Due to under-reporting of Aboriginality to the PDC, it is likely that the true numbers are about one-and-a-half times higher than shown. The level of under-reporting varies between Local Health Districts (Table 69). Data not shown for cell counts less than five. 


\section{Smoking in pregnancy}

In 2009, 50.5\% of Aboriginal or Torres Strait Islander mothers reported smoking at some time during pregnancy, compared to $55.3 \%$ in 2005 . This compares with $10.5 \%$ of non-Aboriginal or Torres Strait Islander mothers who reported smoking at some time during pregnancy in 2009.
Smoking in the second half of pregnancy poses the greatest risk to the health of both mother and baby. In 2009, 47.9\% of Aboriginal or Torres Strait Islander mothers reported smoking in the second half of pregnancy. This percentage varied from $14.3 \%$ in the Northern Sydney Local Health District to $58.8 \%$ in the Southern NSW Local Health District (Figure 3).

Figure 3. Smoking in the second half of pregnancy among Aboriginal and Torres Strait Islander mothers by amount smoked and Local Health District of residence, NSW 2009\#

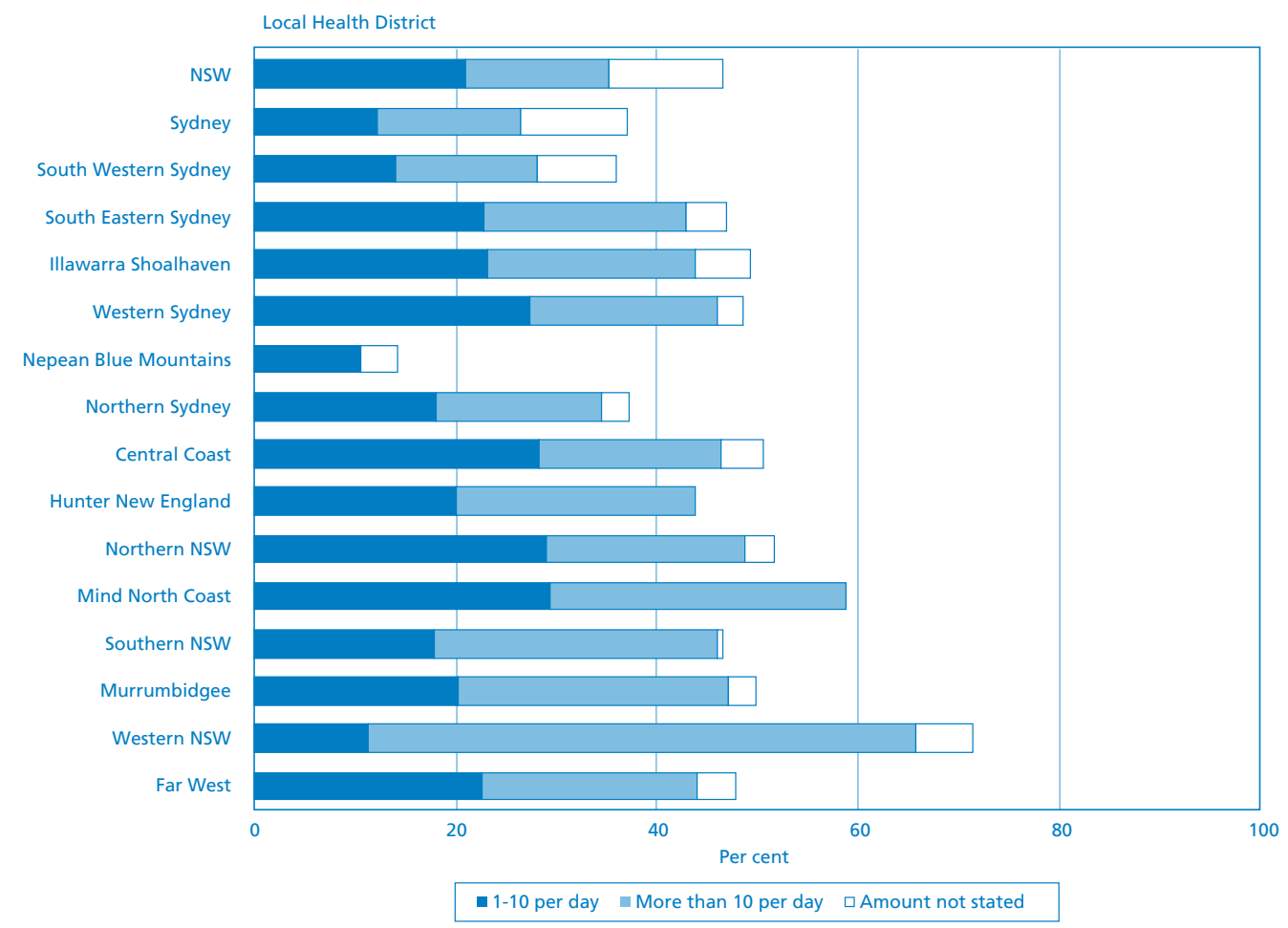

Source: NSW Perinatal Data Collection (HOIST). Centre for Epidemiology and Research. NSW Ministry of Health.

\# Due to under-reporting of Aboriginal mothers on the PDC, it is likely that the true numbers area about one and a half times higher than shown. The level of reporting varies between Local Health Districts (Table 69).

\section{Medical conditions and obstetric complications}

In 2009, there was a lower rate of gestational diabetes and pregnancy-induced hypertension and a higher rate of diabetes mellitus reported among Aboriginal or Torres Strait Islander mothers compared with non-Aboriginal or Torres Strait Islander mothers (Table 79). The number of Aboriginal or
Torres Strait Islander mothers with medical conditions and obstetric complications reported to the PDC is low, even after taking into account under-reporting of Aboriginal mothers. The low numbers may be due to under-detection and/or under-reporting.

Table 79. Maternal medical conditions and obstetric complications among Aboriginal and non-Aboriginal mothers, NSW 2009\#

\begin{tabular}{|c|c|c|c|c|c|c|c|c|}
\hline \multirow[t]{3}{*}{ Condition } & \multicolumn{8}{|c|}{ Aboriginality } \\
\hline & \multicolumn{2}{|c|}{$\begin{array}{l}\text { Aboriginal/ Torres } \\
\text { Strait islander }\end{array}$} & \multicolumn{2}{|c|}{$\begin{array}{l}\text { Non-Aboriginal/ Torres } \\
\text { Strait islander }\end{array}$} & \multicolumn{2}{|c|}{ Not stated } & \multicolumn{2}{|c|}{ TOTAL } \\
\hline & No. & $\%$ & No. & $\%$ & No. & $\%$ & No. & $\%$ \\
\hline Diabetes mellitus & 22 & 0.8 & 503 & 0.5 & 0 & 0.0 & 525 & 0.6 \\
\hline Gestational diabetes & 96 & 3.3 & 5050 & 5.5 & 11 & 6.3 & 5157 & 5.4 \\
\hline Essential hypertension & 26 & 0.9 & 730 & 0.8 & 0 & 0.0 & 756 & 0.8 \\
\hline Pregnancy-induced hypertension & 160 & 5.5 & 5987 & 6.5 & 6 & 3.4 & 6153 & 6.5 \\
\hline TOTAL & 2904 & 100.0 & 91963 & 100.0 & 176 & 100.0 & 95043 & 100.0 \\
\hline
\end{tabular}




\section{Labour and birth}

The rate of spontaneous onset of labour among Aboriginal or Torres Strait Islander mothers fell from $65.2 \%$ in 2005 to $63.8 \%$ in 2009 (Table 80), while the rate of induction of labour was stable at about $23 \%$ to $24 \%$. The rate of induction of labour among Aboriginal or Torres Strait Islander mothers was slightly lower than the rate of $25.8 \%$ reported among non-Aboriginal or Torres Strait Islander mothers in 2009.
Between 2005 and 2009, the rate of normal vaginal birth fell from $70.4 \%$ to $63.8 \%$. The caesarean section rate rose from $23.3 \%$ to $24.3 \%$ (Table 81 ). The rate of instrumental delivery rose slightly from $5.4 \%$ to $6.5 \%$, and about $1 \%$ of all births were vaginal breech births.

Table 80. Labour onset for Aboriginal and Torres Strait Islander mothers, NSW 2005-2009\#

\begin{tabular}{|c|c|c|c|c|c|c|c|c|c|c|}
\hline \multirow[t]{3}{*}{ Labour onset } & \multicolumn{10}{|c|}{ Year } \\
\hline & \multicolumn{2}{|c|}{2005} & \multicolumn{2}{|c|}{2006} & \multicolumn{2}{|c|}{2007} & \multicolumn{2}{|c|}{2008} & \multicolumn{2}{|c|}{2009} \\
\hline & No. & $\%$ & No. & $\%$ & No. & $\%$ & No. & $\%$ & No. & $\%$ \\
\hline Spontaneous & 1613 & 65.2 & 1728 & 66.2 & 1884 & 65.2 & 1966 & 66.1 & 1852 & 63.8 \\
\hline No labour\#\# & 277 & 11.2 & 329 & 12.6 & 315 & 10.9 & 339 & 11.4 & 357 & 12.3 \\
\hline Induced & 584 & 23.6 & 552 & 21.1 & 688 & 23.8 & 669 & 22.5 & 695 & 23.9 \\
\hline Not stated & 0 & 0.0 & 2 & 0.1 & 1 & 0.0 & 2 & 0.1 & 0 & 0.0 \\
\hline TOTAL & 2474 & 100.0 & 2611 & 100.0 & 2888 & 100.0 & 2976 & 100.0 & 2904 & 100.0 \\
\hline
\end{tabular}

Table 81. Type of birth among Aboriginal and Torres Strait Islander mothers, NSW 2005-2009\#

\begin{tabular}{|c|c|c|c|c|c|c|c|c|c|c|}
\hline \multirow[t]{3}{*}{ Labour onset } & \multicolumn{10}{|c|}{ Year } \\
\hline & \multicolumn{2}{|c|}{2005} & \multicolumn{2}{|c|}{2006} & \multicolumn{2}{|c|}{2007} & \multicolumn{2}{|c|}{2008} & \multicolumn{2}{|c|}{2009} \\
\hline & No. & $\%$ & No. & $\%$ & No. & $\%$ & No. & $\%$ & No. & $\%$ \\
\hline Normal vaginal & 1741 & 70.4 & 1868 & 71.5 & 2015 & 69.8 & 2074 & 69.7 & 1984 & 68.3 \\
\hline Forceps & 42 & 1.7 & 46 & 1.8 & 42 & 1.5 & 75 & 2.5 & 64 & 2.2 \\
\hline Vacuum extraction & 91 & 3.7 & 84 & 3.2 & 121 & 4.2 & 124 & 4.2 & 126 & 4.3 \\
\hline Vaginal breech & 24 & 1.0 & 17 & 0.7 & 27 & 0.9 & 25 & 0.8 & 23 & 0.8 \\
\hline Elective caesarean section & 277 & 11.2 & 329 & 12.6 & 315 & 10.9 & 339 & 11.4 & 357 & 12.3 \\
\hline Emergency caesarean section\#\# & 299 & 12.1 & 259 & 9.9 & 365 & 12.6 & 336 & 11.3 & 350 & 12.1 \\
\hline Not stated & 0 & 0.0 & 8 & 0.3 & 3 & 0.1 & 3 & 0.1 & 0 & 0.0 \\
\hline TOTAL & 2474 & 100.0 & 2611 & 100.0 & 2888 & 100.0 & 2976 & 100.0 & 2904 & 100.0 \\
\hline
\end{tabular}




\section{Birth weight}

Since 2005, the rate of low birth weight (less than 2,500 grams) in Aboriginal or Torres Strait Islander babies has been over $10 \%$ and was $10.8 \%$ in 2009 (Table 82). This is about twice the rate for babies born to non-Aboriginal or Torres
Strait Islander mothers, which was 5.8\% in 2009. In 2009, the largest number of low birth weight babies was in the Hunter New England Local Health District (Table 83).

\section{Table 82. Weight of Aboriginal and Torres Strait Islander babies, NSW 2005-2009\#}

\begin{tabular}{|c|c|c|c|c|c|c|c|c|c|c|}
\hline \multirow[t]{3}{*}{ Birth weight (grams) } & \multicolumn{10}{|c|}{ Year } \\
\hline & \multicolumn{2}{|c|}{2005} & \multicolumn{2}{|c|}{2006} & \multicolumn{2}{|c|}{2007} & \multicolumn{2}{|c|}{2008} & \multicolumn{2}{|c|}{2009} \\
\hline & No. & $\%$ & No. & $\%$ & No. & $\%$ & No. & $\%$ & No. & $\%$ \\
\hline Less than 1,000 & 36 & 1.4 & 26 & 1.0 & 57 & 1.9 & 39 & 1.3 & 36 & 1.2 \\
\hline $1,000-1,499$ & 20 & 0.8 & 29 & 1.1 & 23 & 0.8 & 36 & 1.2 & 22 & 0.8 \\
\hline $1,500-2,499$ & 257 & 10.3 & 273 & 10.3 & 276 & 9.4 & 272 & 9.0 & 259 & 8.8 \\
\hline $2,500+$ & 2193 & 87.5 & 2318 & 87.5 & 2563 & 87.6 & 2665 & 88.4 & 2610 & 89.0 \\
\hline Not stated & 1 & 0.0 & 3 & 0.1 & 7 & 0.2 & 3 & 0.1 & 4 & 0.1 \\
\hline TOTAL & 2507 & 100.0 & 2649 & 100.0 & 2926 & 100.0 & 3015 & 100.0 & 2931 & 100.0 \\
\hline
\end{tabular}

Source: NSW Perinatal Data Collection (HOIST). Centre for Epidemiology and Research, NSW Ministry of Health.

\# Due to under-reporting of Aboriginality to the PDC, it is likely that the true numbers are about two times higher than shown.

Table 83. Weight of Aboriginal and Torres Strait Islander babies by Local Health District of residence, NSW 2009\#

\begin{tabular}{|c|c|c|c|c|c|c|c|c|}
\hline \multirow[t]{3}{*}{ Local Health District } & \multicolumn{8}{|c|}{ Birth weight (grams) } \\
\hline & \multicolumn{2}{|c|}{$<2,500$} & \multicolumn{2}{|c|}{$2,500+$} & \multicolumn{2}{|c|}{ Not stated } & \multicolumn{2}{|c|}{ TOTAL } \\
\hline & No. & $\%$ & No. & $\%$ & No. & $\%$ & No. & $\%$ \\
\hline Sydney & 15 & 14.0 & 92 & 86.0 & 0 & 0.0 & 107 & 100.0 \\
\hline South Western Sydney & 16 & 9.2 & 156 & 90.2 & 1 & 0.6 & 173 & 100.0 \\
\hline South Eastern Sydney & 6 & 9.2 & 59 & 90.8 & 0 & 0.0 & 65 & 100.0 \\
\hline Illawarra Shoalhaven & 14 & 8.2 & 157 & 91.8 & 0 & 0.0 & 171 & 100.0 \\
\hline Western Sydney & 28 & 13.7 & 176 & 86.3 & 0 & 0.0 & 204 & 100.0 \\
\hline Nepean Blue Mountains & 13 & 11.3 & 102 & 88.7 & 0 & 0.0 & 115 & 100.0 \\
\hline Northern Sydney & - & - & - & - & - & - & 28 & 100.0 \\
\hline Central Coast & 10 & 9.1 & 100 & 90.9 & 0 & 0.0 & 110 & 100.0 \\
\hline Hunter New England & 82 & 12.4 & 579 & 87.5 & 1 & 0.2 & 662 & 100.0 \\
\hline Northern NSW & 21 & 10.3 & 183 & 89.7 & 0 & 0.0 & 204 & 100.0 \\
\hline Mid North Coast & 29 & 12.2 & 207 & 87.3 & 1 & 0.4 & 237 & 100.0 \\
\hline Southern NSW & 5 & 7.4 & 63 & 92.6 & 0 & 0.0 & 68 & 100.0 \\
\hline Murrumbidgee & 12 & 7.6 & 144 & 91.7 & 1 & 0.6 & 157 & 100.0 \\
\hline Western NSW & 56 & 10.1 & 501 & 89.9 & 0 & 0.0 & 557 & 100.0 \\
\hline Far West & - & - & - & - & - & - & 35 & 100.0 \\
\hline Other-not stated & 5 & 13.2 & 33 & 86.8 & 0 & 0.0 & 38 & 100.0 \\
\hline TOTAL & 317 & 10.8 & 2610 & 89.0 & 4 & 0.1 & 2931 & 100.0 \\
\hline
\end{tabular}




\section{Gestational age}

Since 2005 , over $10 \%$ of Aboriginal or Torres Strait Islander babies have been premature (less than 37 weeks gestation) (Table 84 ). The rate of prematurity was $10.5 \%$ in $2009-$ compared with a rate of $7.2 \%$ for babies born to
non-Aboriginal or Torres Strait Islander mothers. In 2009, the largest number of premature babies was born in the Hunter New England Local Health District (Table 85).

Table 84. Gestational age of Aboriginal and Torres Strait Islander babies, NSW 2005-2009\#

\begin{tabular}{|c|c|c|c|c|c|c|c|c|c|c|}
\hline \multirow[t]{3}{*}{ Gestational age (weeks) } & \multicolumn{10}{|c|}{ Year } \\
\hline & \multicolumn{2}{|c|}{2005} & \multicolumn{2}{|c|}{2006} & \multicolumn{2}{|c|}{2007} & \multicolumn{2}{|c|}{2008} & \multicolumn{2}{|c|}{2009} \\
\hline & No. & $\%$ & No. & $\%$ & No. & $\%$ & No. & $\%$ & No. & $\%$ \\
\hline$<28$ & 30 & 1.2 & 25 & 0.9 & 56 & 1.9 & 44 & 1.5 & 39 & 1.3 \\
\hline $28-31$ & 27 & 1.1 & 26 & 1.0 & 28 & 1.0 & 40 & 1.3 & 26 & 0.9 \\
\hline $32-36$ & 244 & 9.7 & 235 & 8.9 & 289 & 9.9 & 284 & 9.4 & 244 & 8.3 \\
\hline $37-41$ & 2180 & 87.0 & 2330 & 88.0 & 2518 & 86.1 & 2622 & 87.0 & 2589 & 88.3 \\
\hline $42+$ & 26 & 1.0 & 31 & 1.2 & 32 & 1.1 & 24 & 0.8 & 33 & 1.1 \\
\hline Not stated & 0 & 0.0 & 2 & 0.1 & 3 & 0.1 & 1 & 0.0 & 0 & 0.0 \\
\hline TOTAL & 2507 & 100.0 & 2649 & 100.0 & 2926 & 100.0 & 3015 & 100.0 & 2931 & 100.0 \\
\hline
\end{tabular}

Table 85. Gestational age of Aboriginal and Torres Strait Islander babies by Local Health District of residence, NSW 2009\#

\begin{tabular}{|c|c|c|c|c|c|c|}
\hline \multirow[t]{3}{*}{ Local Health District } & \multicolumn{6}{|c|}{ Gestational age (weeks) } \\
\hline & \multicolumn{2}{|c|}{ Less than 37} & \multicolumn{2}{|c|}{$37+$} & \multicolumn{2}{|c|}{ TOTAL } \\
\hline & No. & $\%$ & No. & $\%$ & No. & $\%$ \\
\hline Sydney & 9 & 8.4 & 98 & 91.6 & 107 & 100.0 \\
\hline South Western Sydney & 15 & 8.7 & 158 & 91.3 & 173 & 100.0 \\
\hline South Eastern Sydney & 8 & 12.3 & 57 & 87.7 & 65 & 100.0 \\
\hline Illawarra Shoalhaven & 16 & 9.4 & 155 & 90.6 & 171 & 100.0 \\
\hline Western Sydney & 24 & 11.8 & 180 & 88.2 & 204 & 100.0 \\
\hline Nepean Blue Mountains & 15 & 13.0 & 100 & 87.0 & 115 & 100.0 \\
\hline Northern Sydney & - & - & - & - & 28 & 100.0 \\
\hline Central Coast & 7 & 6.4 & 103 & 93.6 & 110 & 100.0 \\
\hline Hunter New England & 90 & 13.6 & 572 & 86.4 & 662 & 100.0 \\
\hline Northern NSW & 15 & 7.4 & 189 & 92.6 & 204 & 100.0 \\
\hline Mid North Coast & 25 & 10.5 & 212 & 89.5 & 237 & 100.0 \\
\hline Southern NSW & - & - & - & - & 68 & 100.0 \\
\hline Murrumbidgee & 12 & 7.6 & 145 & 92.4 & 157 & 100.0 \\
\hline Western NSW & 60 & 10.8 & 497 & 89.2 & 557 & 100.0 \\
\hline Far West & - & - & - & - & 35 & 100.0 \\
\hline Other-not stated & 4 & 10.5 & 34 & 89.5 & 38 & 100.0 \\
\hline TOTAL & 309 & 10.5 & 2622 & 89.5 & 2931 & 100.0 \\
\hline
\end{tabular}

\section{Apgar score}

In 2009, 3.1\% of Aboriginal or Torres Strait Islander babies had an Apgar score less than seven (Table 86), slightly higher than the rate of $2.0 \%$ for babies born to non-Aboriginal or Torres Strait Islander mothers.

Table 86. Apgar score of Aboriginal and Torres Strait Islander babies, NSW 2005-2009\#

\begin{tabular}{|c|c|c|c|c|c|c|c|c|c|c|}
\hline \multirow[t]{3}{*}{ Apgar score at 5 minutes } & \multicolumn{10}{|c|}{ Year } \\
\hline & \multicolumn{2}{|c|}{2005} & \multicolumn{2}{|c|}{2006} & \multicolumn{2}{|c|}{2007} & \multicolumn{2}{|c|}{2008} & \multicolumn{2}{|c|}{2009} \\
\hline & No. & $\%$ & No. & $\%$ & No. & $\%$ & No. & $\%$ & No. & $\%$ \\
\hline $0-4$ & 42 & 1.7 & 36 & 1.4 & 73 & 2.5 & 62 & 2.1 & 53 & 1.8 \\
\hline $5-6$ & 33 & 1.3 & 24 & 0.9 & 42 & 1.4 & 45 & 1.5 & 39 & 1.3 \\
\hline $7+$ & 2416 & 96.4 & 2571 & 97.1 & 2794 & 95.5 & 2886 & 95.7 & 2819 & 96.2 \\
\hline Not stated & 16 & 0.6 & 18 & 0.7 & 17 & 0.6 & 22 & 0.7 & 20 & 0.7 \\
\hline TOTAL & 2507 & 100.0 & 2649 & 100.0 & 2926 & 100.0 & 3015 & 100.0 & 2931 & 100.0 \\
\hline
\end{tabular}




\section{Infant feeding}

In 2009, $62.4 \%$ of babies born to Aboriginal or Torres Strait Islander mothers were fully breastfed at discharge from hospital, $4.5 \%$ were partially breastfed and $31.3 \%$ received infant formula only (Table 87 ). This compares with $80.6 \%$ of babies born to non-Aboriginal or Torres Strait Islander mothers who were fully breastfed in 2009. Rates of full breastfeeding ranged from $51.0 \%$ in the Western Sydney Local Health District to $92.9 \%$ in the Northern Sydney Local Health District.

Table 87. Infant feeding among Aboriginal and Torres Strait Islander liveborn babies by Local Health District of residence, NSW 2009\#

\begin{tabular}{|c|c|c|c|c|c|c|c|c|c|c|}
\hline \multirow[t]{3}{*}{ Local Health District } & \multicolumn{10}{|c|}{ Infant feeding } \\
\hline & \multicolumn{2}{|c|}{$\begin{array}{l}\text { Full breast- } \\
\text { feeding }\end{array}$} & \multicolumn{2}{|c|}{$\begin{array}{l}\text { Any breast- } \\
\text { feeding }\end{array}$} & \multicolumn{2}{|c|}{$\begin{array}{c}\text { Infant } \\
\text { formula only }\end{array}$} & \multicolumn{2}{|c|}{ Not stated } & \multicolumn{2}{|c|}{ TOTAL } \\
\hline & No. & $\%$ & No. & $\%$ & No. & $\%$ & No. & $\%$ & No. & $\%$ \\
\hline Sydney & 64 & 59.8 & 13 & 12.1 & 29 & 27.1 & 1 & 0.9 & 107 & 100.0 \\
\hline South Western Sydney & 90 & 52.0 & 17 & 9.8 & 64 & 37.0 & 2 & 1.2 & 173 & 100.0 \\
\hline South Eastern Sydney & 48 & 73.8 & - & 3.1 & 14 & 21.5 & - & - & 65 & 100.0 \\
\hline Illawarra Shoalhaven & 109 & 63.7 & - & 2.3 & 53 & 31.0 & - & - & 171 & 100.0 \\
\hline Western Sydney & 104 & 51.0 & 9 & 4.4 & 87 & 42.6 & 4 & 2.0 & 204 & 100.0 \\
\hline Nepean Blue Mountains & 65 & 56.5 & 5 & 4.3 & 43 & 37.4 & 2 & 1.7 & 115 & 100.0 \\
\hline Northern Sydney & 26 & 92.9 & - & - & - & - & - & - & 28 & 100.0 \\
\hline Central Coast & 76 & 69.1 & - & - & 29 & 26.4 & - & - & 110 & 100.0 \\
\hline Hunter New England & 410 & 61.9 & 30 & 4.5 & 205 & 31.0 & 17 & 2.6 & 662 & 100.0 \\
\hline Northern NSW & 140 & 68.6 & 13 & 6.4 & 47 & 23.0 & 4 & 2.0 & 204 & 100.0 \\
\hline Mid North Coast & 182 & 76.8 & 7 & 3.0 & 44 & 18.6 & 4 & 1.7 & 237 & 100.0 \\
\hline Southern NSW & 51 & 75.0 & 0 & 0.0 & 16 & 23.5 & 1 & 1.5 & 68 & 100.0 \\
\hline Murrumbidgee & 95 & 60.5 & - & - & 57 & 36.3 & - & - & 157 & 100.0 \\
\hline Western NSW & 319 & 57.3 & 21 & 3.8 & 210 & 37.7 & 7 & 1.3 & 557 & 100.0 \\
\hline Far West & 21 & 60.0 & 5 & 14.3 & 9 & 25.7 & 0 & 0.0 & 35 & 100.0 \\
\hline Other-not stated & 28 & 73.7 & 0 & 0.0 & 7 & 18.4 & 3 & 7.9 & 38 & 100.0 \\
\hline TOTAL & 1828 & 62.4 & 131 & 4.5 & 916 & 31.3 & 56 & 1.9 & 2931 & 100.0 \\
\hline
\end{tabular}

Source: NSW Perinatal Data Collection (HOIST). Centre for Epidemiology and Research, NSW Ministry of Health.

\# Due to under-reporting of Aboriginality to the PDC, it is likely that the true numbers are about two times higher than shown. The level of under-reporting varies between Local Health Districts (Table 69). Data not shown for cell counts less than five.

\section{Perinatal mortality}

Since 2005, the perinatal mortality rate among Aboriginal or Torres Strait Islander babies has varied from 12.1 to 22.6 per 1,000 births (Table 88). The rate of 12.6 per 1,000 in 2009 is substantially higher than the rate of 8.5 per 1,000 experienced by babies born to non-Aboriginal or Torres Strait Islander mothers.

Table 88. Perinatal deaths among Aboriginal and Torres Strait Islander babies, NSW 2005-2009\#

\begin{tabular}{|c|c|c|c|c|c|c|c|c|c|c|}
\hline \multirow[t]{3}{*}{ Perinatal deaths } & \multicolumn{10}{|c|}{ Year } \\
\hline & \multicolumn{3}{|c|}{2005} & \multicolumn{2}{|l|}{2006} & \multicolumn{2}{|l|}{2007} & \multicolumn{2}{|l|}{2008} & 2009 \\
\hline & No. & Rate/ 1,000 & No. & Rate/ 1,000 & No. & Rate/ 1,000 & No. & Rate/ 1,000 & No. & Rate/ 1,000 \\
\hline Stillbirth & 22 & 8.8 & 16 & 6.0 & 41 & 14.0 & 34 & 11.3 & 27 & 9.2 \\
\hline Neonatal death & 16 & 6.4 & 16 & 6.0 & 25 & 8.5 & 12 & 4.0 & 10 & 3.4 \\
\hline TOTAL & 38 & 15.2 & 32 & 12.1 & 66 & 22.6 & 46 & 15.3 & 37 & 12.6 \\
\hline
\end{tabular}

Source: NSW Perinatal Data Collection (HOIST). Centre for Epidemiology and Research, NSW Ministry of Health.

\# Perinatal deaths include deaths reported to the PDC only. As the PDC form is completed at discharge or transfer of the baby, deaths occurring after this time may not be reported to the MDC. Due to under-reporting of Aboriginality to the PDC, it is likely that the true numbers are about two times higher than shown. 